\section{The pentaquark:}

\section{a new kind of}

\section{elementary particle?}

Klaus Goeke ', Hyun-Chul Kim ${ }^{2}$, Michat Praszatowicz ${ }^{3}$

${ }^{\prime}$ Institut für Theoretische Physik II, Ruhr-Universität Bochum, D-44780 Bochum, Germany,

${ }^{2}$ Dep. of Physics, and Nuclear Physics \& Radiation Technology Institute (NuRI), Pusan National University, 609-735 Pusan, Republic of Korea,

${ }^{3}$ M. Smoluchowski Institute of Physics, Jagellonian University, ul. Reymonta 4, 30-059 Kraków, Poland.

espite intensive experimental studies for half a century and theoretical work based on Quantum Chromodynamics (QCD) in the last 30 years, our understanding of nucleon structure is still far from being complete. This is reflected by the several competing models of nucleons or, more broadly, light baryons, as e.g. various constituent quark models with gluonic or chiral forces, or topological and non-topological solitonic models. There are also sum-rule approaches and lattice gauge calculations, which are less model dependent but not always reliable. This situation is unsatisfactory. Nucleons are the main building blocks of matter around us, they provide the mass for the baryonic (i.e. visible) matter of the universe, and we need to understand their structure and their dynamics.

In this situation the prediction and discovery of the narrow baryonic resonance $\Theta^{+}$with strangeness of +1 , i.e. containing one excess strange anti-quark, may prove to be extremely important, since it perhaps indicates the existence of a new class of baryons, and this may shed a completely new light on the present models for baryon structure. Actually the $\Theta^{+}$has a mass of $1530 \mathrm{MeV}$, compared e.g. to $938 \mathrm{MeV}$ for the nucleon, and a decay width of the order of $1 \mathrm{MeV}$, which is two orders of magnitude smaller than expected for baryons in this energy region. It has in 2002 and 2003 been identified by several groups [1] using different reaction processes. Such a state is extremely exciting because it is unambiguously exotic in the sense that it cannot be a simple three-quark state. These experiments have been triggered by predictions of mass and decay width in the chiral quark soliton model ( $\chi Q S M)$ by Diakonov, Petrov and Polyakov [2] in St.Petersburg and Bochum. An earlier estimate of the mass in the soliton approach of the Skyrme model was given by Praszatowicz [3] in Krakow. The discovery of $\Theta^{+}$together with the accurate prediction of Ref.[2] have initiated considerable theoretical activity. The discussion got even very heated since several other experiments in the last year, mostly of higher energy, did not show any evidence of the $\Theta^{+}$, see refs. [4]. Besides the $\Theta^{+}$there is perhaps an observation of an exotic $\Xi_{\overline{10}}$ state at $1860 \mathrm{MeV}$ by the NA49 experiment at CERN [5], though it is still under debate. Much of the theoretical activity has been aimed at understanding the structure of these exotic states, $\Theta^{+}$in particular. Besides using the chiral solitonic approach the most common treatment of this problem has been based on extensions and variants of the constituent quark model [7] and of the Skyrme model [8]. While these approaches are all interesting, they all are also highly model-dependent and it is difficult to assess in an a priori way their validity.

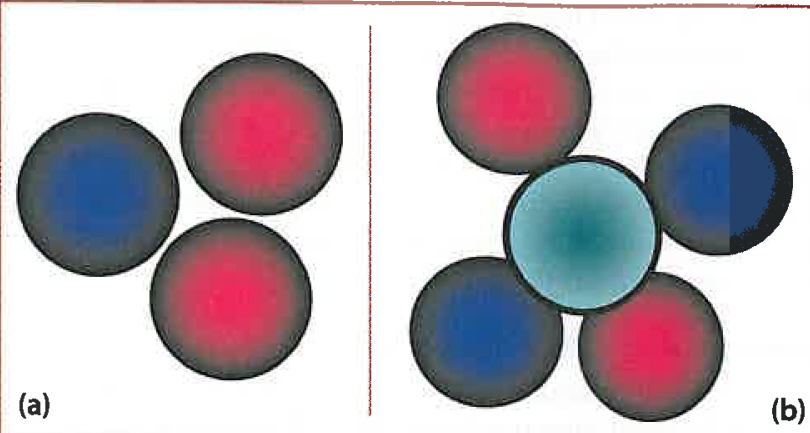

Fig. 1: (a)The structure of the proton with two up-and one down-quark (uud). (b)The structure of the $\Theta^{+}$with two upquarks, two down-quarks and one strange anti-quark (uudd $\bar{s}$ ).

The analysis based on the SU(3) chiral soliton model [2] appears different from other treatments of the $\Theta^{+}$structure in a number of ways: I) Exotic SU(3) representations containing exotic baryonic states are naturally accommodated within the chiral soliton models [3]. II) The soliton approach was used to predict exotic states by linking their properties to the known baryons in octet and decuplet of SU(3) flavour. In contrast to the other treatments it preceded experimental discovery by many years. III) Despite some freedom as far as model parameters are concerned the predictions of the mass were very accurate $[2,3]$. IV) The width was predicted to be very small [2], which is consistent with the widths presently observed [9].

In the present paper we will in the first part review the quark model of baryons and give some historical background. Then we discuss the deficiencies of the quark model, consider spontaneously broken chiral symmetry, and focus our attention on the solitonic (mean field) approach to the $\Theta^{+}$and the anti-decuplet in the framework of a relativistic quantum field theory [6]. A discussion of the decay width and a report on the discovery of $\Theta^{+}$ finalizes the paper.

\section{The quark model: history and presence}

In 1963 Gell-Mann and Zweig suggested a model for the nucleon and the light baryons which was based on the concept of group theory. They were able to classify the baryons by the quantum numbers of isospin $\mathrm{T}, T_{3}$ and hypercharge $Y$, characteristic of the multiplets of the symmetry group SU(3)-flavour. The model was formulated in terms of quarks, that constituted the fundamental representation of $\mathrm{SU}(3)$. The model turned out to be highly successful and allowed Gell-Mann to predict the existence of a new particle, the $\Omega$-, which was rather soon identified experimentally. The model was then extended dynamically involving phenomenological potentials, in which the quarks moved, or appropriate quark-quark interactions, which in the modern terminology consisted of confining potentials and gluon and/or meson exchange forces. The forces and quark masses were adjusted to observables of the baryons as masses, magnetic moments, radii, etc. In the end the following picture emerged: The nucleon and the light baryons consist of three quarks with spin $=1 / 2$ and with flavours up, down and strange. These have fractional charges $Q=2 e / 3$, $-e / 3,-e / 3$ and masses of about $350 \mathrm{MeV}$ for up- and down-quarks and for the strange quark about $100-200 \mathrm{MeV}$ heavier. For example the proton consists in this scheme of two up-quarks and one down-quark (uud, see fig.la). The baryons can be arranged in octets and decuplets of the $S U(3)$ flavour group, characterized by quantum numbers of isospin T, $T_{3}$ and hypercharge $Y$ and combined with an antisymmetric colour structure. All attempts to 
find structures not belonging to this scheme, e.g. containing another number of quarks or besides quarks also a few anti-quarks, were in vain. This historical circumstance led to the wide-spread belief that 'exotic' baryons, which cannot be constructed of three quarks and belong to higher $S U(3)$ multiplets, do not exist. This belief might be now discredited with the discovery of the $\Theta^{+}$. It has the quark structure $u u d d \bar{s}$ and hence consists of five (Greek: penta) quarks rather than three, see fig.lb.

\section{Concept of constituant quarks}

In the 1970's Quantum Chromodynamics (QCD) was formulated as a relativistic quantum field theory of the strong interaction involving quarks as particles and gluons as interacting field quanta. As far as the up-, down- and strange sector was concerned, these quarks have small masses of $m_{u} \simeq 4 \mathrm{MeV}, m_{d} \simeq 7 \mathrm{MeV}$ (and $m_{s} \simeq 150 \mathrm{MeV}$ ) and are so called current quarks to be distinguished from constituent quarks in the quark models of about $350 \mathrm{MeV}$. For massless quarks (and even $150 \mathrm{MeV}$ is nearly massless) the QCD-Lagrangian shows chiral symmetry. This means that in the QCD-Lagrangian right-handed quarks do not interact with left-handed ones and vice versa. However this symmetry is not realized in nature but is spontaneously broken. This effect occurs analogously in ferromagnetism, where the forces which couple the electronic spins and hence the Hamiltonian of the system are rotationally invariant, whereas in the ground state the spins are aligned in some definite direction resulting in a nonzero magnetization and violation of the rotational symmetry.

For the structure of light hadrons this spontaneous breaking of chiral symmetry is probably the most important mechanism. This effect has its origin in the zero-point quantum fluctuations of the gluon field in the vacuum of QCD. Peculiar for QCD is that there are specific large fluctuations called instantons with average size and average separation of roughly $1 / 3 \mathrm{fm}$ and $1 \mathrm{fm}$, respectively. If light quarks propagate through this fluctuating gluon vacuum, "hopping" from one randomly situated instanton fluctuation to another, they behave like electrons in a lattice structure, i.e. they gain an effective mass which in the case of the quarks is a large dynamical momentum dependent mass $M(p)$ serving of equal importance as the quark-pion coupling constant. This dynamical mass is the key for understanding the properties of light hadrons. Its value at zero momentum, $M(0) \simeq 350 \mathrm{MeV}$, is what is usually called the "constituent" quark mass (to be distinguished from the small input "current" quark masses of the QCD Lagrangian). Thus spontaneous breaking of chiral symmetry results in the end in the following effect: The almost massless $u, d, s$ current quarks acquire the dynamical momentum-dependent masses $M u, d, s(p)$ (constituent masses) and this is simultaneously connected with the occurrence of massless Goldstone bosons which are in nature the light (nearly massless) pseudoscalar mesons $\pi, K, \eta$.

The standard belief is that a linear confining potential provides the mechanism to bind constituent quarks to baryons and mesons. The confining potential is supposed to show some $1 / r$ dependence for small distances and a linear rise $r$ at large ones. However, in the real world with very light pions this cannot be correct because while increasing the distance between two quarks it is energetically favorable to create quark-antiquark pairs from the vacuum and to screen the confinement potential. The screening is so strong that in the end all that is left are constituent quarks with a dynamical momentum dependent mass $M(p)$ interacting with a mean field with the quantum numbers of the pseudoscalar Goldstone bosons, i.e. $\pi, K$ and $\eta$. Actually there is not much freedom in formulating this in terms of a field theory since the interaction of pseudoscalar mesons with constituent quarks is to a large extent dictated by the chiral symmetry of QCD [10] yielding a well defined and more or less unique lowmomenta effective theory.

\section{Relativistic mean field approach to the nucleon}

The low-momenta quark-pion effective theory described above is a big step forward, as compared to the original formulation of QCD: it operates with the adequate degrees of freedom, namely the dynamically-massive quarks and the light pseudoscalar meson field [6]. The transition to these new degrees of freedom is similar to the transition from Quantum Electrodynamics (i.e. the microscopic theory of the atoms) to the electrons in a material, whose mass is not the original $0.511 \mathrm{MeV}$ but a heavier effective mass, and whose most important interaction at the atomic "low energies" is not the Coulomb (read: gluon) field but rather the phonon (read: pion) exchange. Phonons are collective excitations of atomic lattices, and they are Goldstone bosons associated with the spontaneous breaking of the translational symmetry by the lattice. Pions are collective excitations of the QCD ground state (vacuum), and they are Goldstone bosons associated with the spontaneous breaking of chiral symmetry. Continuing the analogy, the Cooper pairing of electrons in a superconductor is due to phonon exchange that is much stronger than the Coulomb force between electrons (being actually a repulsion). Analogously, the binding of quarks into a nucleon can be explained as due to their interaction with the pion field. Although the corresponding low-momenta effective theory is a great simplification as compared to the microscopic QCD, it is still a strong-coupling relativistic quantum field theory which is solved and applied to light baryons in the chiral quark soliton approach $(\chi Q S M)$ [10] and variants of it [11]. In this approach the quarks with the momentum-dependent mass $M(p)$ move in a self-consistently generated pseudo-scalar mean field similarly to the Hartree-Fock potential in nuclear and atomic physics.

In contrast to the naive quark models, the $\chi Q S M$ is relativistic-invariant. Being such, it necessarily incorporates quark-antiquark admixtures to the nucleon. Quark-antiquark pairs appear in the nucleon on top of the three valence quarks as particle-hole excitations of the Dirac sea distorted by its interaction with the pion mean field [remember: the Dirac sea comprises all the levels with negative single particle energy since they always appear as solutions of the Dirac equation]. In addition there will be vibrations of the multitude of quarks moving in the self-consistent field and the reaction of the self-consistent field to this movement. These excitations should be described by means of relativistic random-phase-approximations, which, however, are not

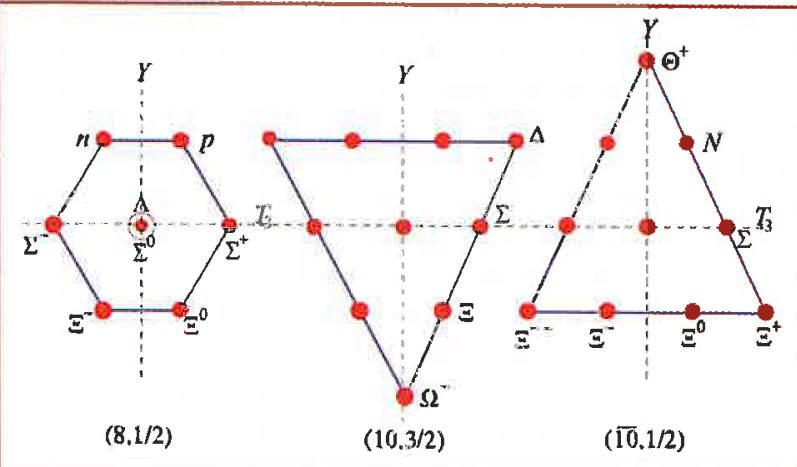

Aig. 2: The lowest baryon multiplets which can be interpreted as rotational states in ordinary and 3-flavour spaces, shown in the hypercharge-isospin $Y-T_{3}$ plane. 


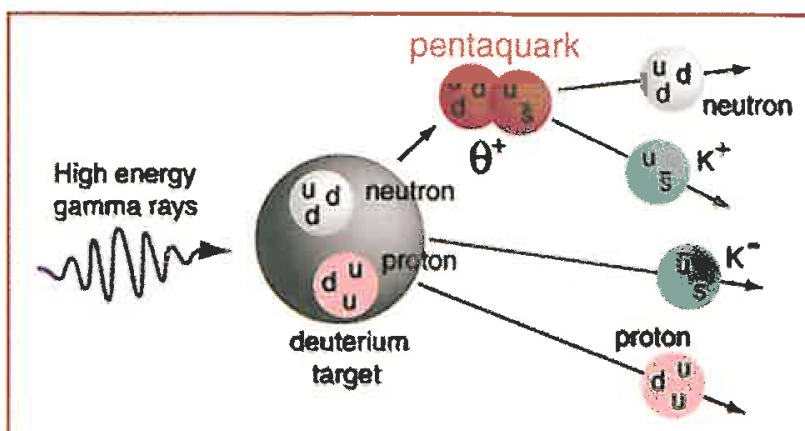

$\triangle$ Fig. 3: The reaction in which the $\Theta^{+}$was found by the HERMES collaboration at DESY

considered in the present context. In fact this mean field approximation with successive quantization of collective rotations in coordinate space and flavour space is known to be very successful, since it has described many properties of light baryons, as e.g. mass splittings and form factors of octet baryons, and parton distributions and generalized parton distributions of the nucleon.

\section{Rotational excitations}

A mean field solution as such does not provide the quantum numbers of the baryon yet. This is well known from nuclear many body physics, where e.g. the Hartree-Fock solution of a deformed nucleus has to be projected by Peierls-Yoccoz techniques on good angular momentum. There the results are multiplets characterized by the total angular momentum $J$ and dimension of $d=2 J+1$. The states inside a multiplet are $\mid J, K>$ with projection $K$ of the angular momentum in the body fixed frame with $K=-J_{, \ldots .}, J$. In the present case for each $J$ the members of each multiplet are characterized by two quantum numbers, i.e. the isospin $T_{3}$ and hypercharge $Y$. The reason lies in the more complicated structure of the symmetry group, which for three flavours of the quarks (up, down, strange) is SU(3). If one denotes the SU(3)-multiplets by their dimension and their angular momentum and parity one obtains $\left(8, \frac{1}{2}^{+}\right),\left(10, \frac{3}{2}^{+}\right),\left(\overline{10}, \frac{1}{2}^{+}\right)$, $\left(27, \frac{3}{2}^{+}\right),\left(27, \frac{1}{2}^{+}\right)$... They are ordered by increasing mass, see Fig. 2. The first two (the octet and the decuplet) are indeed the lowest baryonic multiplets in nature. They are also the only ones whose states can be composed of just three quarks in an orbital s-state, according to the quantum numbers. All other multiplets need explicitly the inclusion of the negative-energy single quark orbits (Dirac sea) and their distortion due to the interaction with the mean field. If one disentangles this distortion by means of the Thouless-Theorem it corresponds to $1 p-1 h$-excitations (or quarkantiquark excitations) with respect to the unperturbed Dirac sea. Hence e.g. the states in the anti-decuplet $\left(\overline{10}, \frac{1}{2}+\right)$ have structure with dominating components uuddū or $u u d d \bar{s}$. Here the $u u d d \bar{u}$ has in $\left(\overline{10}, \frac{1}{2}^{+}\right)$the same quantum numbers as udd of the ordinary octet and hence would fit into a pure 3-quark scheme. On the other hand, the $u u d d \bar{s}$ is truly exotic, since it cannot be formed by three quarks and has no counterpart in the octet.

An interesting question is where to stop in this list of multiplets of the soliton. Apparently for sufficiently high rotational states the rotations become too fast: the centrifugal forces will rip the baryon apart. Also the radiation of pions and kaons by a fast-rotating body is so strong that the widths of the corresponding resonances blow up. Actually one needs to compute their widths in order to make a judgement. If the width turns out to be in the order of hundreds of $\mathrm{MeV}$, one can say that this is where the rotational sequence ceases to exist.
An estimate of the width of the lightest member $\Theta^{+}$of the antidecuplet, shown at the top of the anti-decuplet, gave a surprisingly small result: $\Gamma_{\Theta}<15 \mathrm{MeV}$. This result, obtained in the chiral quark soliton model, immediately gave credibility to the existence of the anti-decuplet and motivated the experimentalists to search for this new baryonic particle.

\section{Prediction and observation of the $\Theta^{+}$}

In 1997 Diakonov, Petrov and Polyakov [2] summarized the results on the antidecuplet obtained in a collaboration between theory groups of the University of Bochum and of the Petersburg Nuclear Physics Institute in Gatchina. There were two striking features: $\Theta^{+}$ had to be relatively light $(1540 \mathrm{MeV})$ and surprisingly narrow $\left(\Gamma_{\theta^{+}}\right.$ $<15 \mathrm{MeV}$ ). This prediction has to be contrasted with naive quark model expectations where such a state should be as heavy as 1.7 $1.9 \mathrm{GeV}$, broader than $100 \mathrm{MeV}$ and of negative parity.

On the experimental side, from 1960's till early 80's there have been intensive searches for exotic $S=+1$ baryons in $1.7-1.9 \mathrm{GeV}$ energy range with no convincing results. After 1986 the Particle Data Group stopped mentioning these searches. The 1992 partial wave analysis of the $K N$ scattering data concluded that there might be broad resonances but, if there were any, they ought to be in a high-mass range. In fact, today we know that those KNdata are consistent with the present finding of the $\Theta^{+}$if this has a width of about one $\mathrm{MeV}$ [9].

In October 2002 T. Nakano [1] from LEPS collaboration reported the first evidence of the new baryon from the $\gamma \mathrm{C}$ reaction. Independently, the DIANA collaboration led by A. Dolgolenko at ITEP, Moscow, looked into their $\mathrm{K}+\mathrm{Xe}$ bubble chamber data. In December 2002 the group reported on the observation of a very narrow $\Theta^{+}$. The next groups, including two CLAS experiments $\left(\gamma d \rightarrow p n K^{+} K^{-}\right.$and $\left.\gamma p \rightarrow n \pi^{+} K^{0} \bar{K}^{0}\right)$ at Jefferson Lab., knew about these two experiments, and gave very important confirmation using various reactions and final states [1]; see fig. 3 for the reaction process at DESY. Later several experiments, mostly of higher energy, announced negative results [4].

It should be noted that all the experimental results (including the negative ones) came from reanalyzing the raw data of old experiments designed originally for other purposes. Recently LEPS (Spring8, Japan) and CLAS (Jlab, USA) have launched dedicated high statistics programs searching for exotic baryons. While the first results from LEPS confirmed the existence of $\Theta^{+}$ in $\gamma d$ photoproduction, the analysis of $\left(\gamma d \rightarrow p n K^{+} \pi^{-} \pi^{0}\right.$ and $\gamma p$ $\rightarrow n \pi^{+} \pi^{-} K^{+}$) reactions at CLAS gave negative results. Preliminary as they are, these findings show our ignorance as far as the production mechanism of $\Theta^{+}$is concerned. Only when the full analysis including different final states is completed one can know whether these high statistics searches will invalidate or confirm the first low statistics sightings announced over the last 3 years. Therefore, one is now looking forward to the forthcoming results, for the issue to be finally resolved.

\section{The decay width of $\Theta^{+}$}

All experiments giving evidence of the $\Theta^{+}$see that it is narrow, the most stringent bound being $\Gamma<9 \mathrm{MeV}$. The indirect estimates [9] show that it can be actually as small as $1 \mathrm{MeV}$ or even less. If correct, $\Theta^{+}$would be the most narrow strongly decaying particle made of light quarks. Any theoretical model of $\Theta^{+}$has to explain the unusually small width first of all.

The Chiral Quark Soliton Model, which motivated the experimentalists, gave an estimate $\Gamma<15 \mathrm{MeV}$ [2]. To understand theoretically the small width it is helpful to go into a coordinate frame where it makes sense even for a relativistic system to talk 
of the number of particles. This is the infinite momentum frame. In this system the baryon wave function falls into separate sectors of the Fock space: three quarks, five quarks (i.e. three quarks plus one quark-antiquark pair), etc. The difference between the ordinary nucleon and the $\Theta^{+}$is that the nucleon has mainly a threequark component and a small five-quark component, while $\Theta^{+}$'s Fock space starts from the five-quark component with small seven-quark admixtures.

For the calculation of the decay width of $\Theta^{+}$it is important that the $\Theta^{+}$decays only into $n+K^{+}$and $p+K^{0}$ and the kaon is not an arbitrary meson but a (nearly massless) Goldstone meson. Hence, it is in some approximation sufficient to evaluate $g_{A}^{\Theta} \rightarrow N K$ as a transition matrix element of the axial charge between $\Theta^{+}$ and the nucleon states. In the infinite-momentum frame the operator of the axial charge does not create or annihilate quarks but only measures the axial charge of the existing quarks. Thus, the matrix element in question is non-zero only between the pentaquark and the five-quark component of the nucleon, and this is known to be small. In addition there is a suppression in the overlap between $\Theta^{+}$and the 5-quark component of the nucleon and there are also some phase space arguments. A crude preliminary estimate shows that the $\Theta^{+}$width can be very small.

\section{Summary}

The discovery of the exotic $\Theta^{+}$with minimal quark structure $u u d d \bar{s}$ may provide a sensation since, if confirmed, it is the first baryonic particle that cannot be composed of three quarks. The chiral quark soliton description of baryons has predicted the mass and an upper limit for the decay width of this particle prior to the experiments and in agreement with the present data. The model corresponds to a relativistic mean field description of the nucleon, where the quarks move in a self-consistent mean field of pionic and kaonic character. It uses an effective chiral Lagrangian based on spontaneously broken chiral symmetry of the QCD. In a natural way the chiral quark soliton model describes the well known lowest two multiplets $\left(8, \frac{1}{2}+\right),\left(10, \frac{3}{2}^{+}\right)$and it predicts two more exotic particles being members of an antidecuplet $\left(\overline{10}, \frac{1}{2}+\right)$ consisting of pentaquarks. The very narrow width of the $\Theta^{+}$can be explained by the small overlap of the 5quark light cone wave function of the $\Theta^{+}$with the small 5-quark light cone component of the wave function of the nucleon.

If confirmed, $\Theta^{+}$will not only be a new kind of subatomic particle but will seriously influence our understanding of the structure of ordinary nucleons. :

\section{About the Authors}

Klaus Goeke, Michał Praszałowicz and Hyun-Chul Kim are professors of theoretical physics at their respective Universities. Both Michat Praszałowicz and Hyun-Chul Kim spend periods in Bochum, as Alexander-von-Humbold-Fellow and Postdoctoral Fellow, respectively. The authors have formed for several years a team with common publications using chiral solitonic models of the structure of the nucleon and light baryons with a focus on the newly discovered pentaquarks, the strange content of the nucleon and model-independent predictions of baryon properties. With their theoretical work they accompany experiments at COSY, ELSA, JLAB, DESY and CERN.

\section{Acknowledgement}

The authors thank D. Diakonov, V. Petrov, M. Polyakov, P. Schweitzer and A. Silva for useful discussions. The paper is partially supported by the SFB/Transregio Bonn-Bochum-Giessen, by the Graduiertenkolleg Dortmund-Bochum, by the
Verbundforschung of the BMFT, by the International Office of DFG (Polish-German), and by the COSY-Juelich project. The work has also been supported by Korean-German DFG-project (F01-2004-000-00102-0).

\section{References}

[1] T. Nakano et al. [LEPS Collaboration], Phys. Rev. Lett. 91, (2003) 012002; V. V. Barmin et al. [DIANA Collaboration], Phys. Atom. Nucl. 66 (2003) 1715 [Yad. Fiz. 66 (2003) 1763]; S. Stepanyan et al. [CLAS Collaboration], Phys. Rev. Lett. 91 (2003) 252001; Phys. Rev. Lett. 92 (2004) 032001 [Erratum-ibid. 92 (2004) 049902]; J. Barth [SAPHIR Collaboration], hep-ex/0307083; A. E. Asratyan, A. G. Dolgolenko and M. A. Kubantsev, Phys. Atom. Nucl. 67 (2004) 682 [Yad. Fiz. 67 (2004) 704]; A. Airapetian et al. [HERMES Collaboration], Phys. Lett. B 585 (2004) 213; A. Aleev et al. [SVD Collaboration], arXiv:hep/ex $0401024 \mathrm{M}$. Abdel-Bary et al. [COSYTOF Collaboration], Phys.Lett.B595:127-134,2004 P. Z. Aslanyan, V.N. Emelyanenko and G. G. Rikhkvitzkaya, arXiv:hep-ex/0403044; S. Chekanov et al. [ZEUS Collaboration], Phys. Lett. B 591 (2004) 7; T. Nakano, QCD-Conference at PKZ, June 20, 2005, priv. Comm..

[2] D. Diakonov, V. Petrov and M. V. Polyakov, Z. Phys. A359 (1997) 305.

[3] M. Praszałowicz, talk at Workshop on Skyrmions and Anomalies, M. Je"abek and M. Praszałowicz eds., World Scientific 1987, page 112 and Phys. Lett. B575 (2003) 234.

[4] J. Z. Bai et al., BES Collaboration, Phys.Rev.D70:012004,2004; K.T. Knöpfle, M. Zavertyaev, T. Zivko for the HERA-B Collaboration, J. Phys.G30:S1363-S1366,2004; G. Lafferty, arXiv:hep-ex/0408064; C. Pinkenburg, PHENIX Collaboration,J.Phys.G30:S1201S1206,2004; S. Schael et al., ALEPH Collaboration, Phys.Lett.B599:1-16,2004; Ming-Jer Wang et al., I. Kravchenko, CDF Collaboration, www.qnp2004.org/; J. Coleman, BABAR Collaboration, arXiv:hep-ex/0502004; R. deVita, CLAS Collaboration, www.phy.ohiou.edu/ hicks/thplus/New/RDeVita-APS05.pdf

[5] C. Alt et al. [NA49 Collaboration], Phys. Rev. Lett. 92 (2004) 042003 [arXiv:hep-ex/0310014];

[6] D. Diakonov, arXiv:hep-ph/0406043.

[7] R. L. Jaffe and F. Wilczek,Phys. Rev. Lett. 91, (2003) 23002;R. L. Jaffe and F. Wilczek, Phys.Rev.D69:114017,2004; F. Stancu and D. O. Riska, Phys. Lett. B. 575, (2003) 242; S. Capstick et al., Phys. Lett. B 570, (2003) 185; B. G. Wyboume, hep-ph/0307170; A. Hoska, Phys. Lett. B 571, (2003) 55; M. Karliner and H. J. Lipkin,hep-ph/0307343; C. E. Carlsonet al., Phys. Lett. B 573, 101 (2003); K. Cheung, hepph/0308176; V. E. Lyubovitskijet al., Phys. Rev. C66 (2002) 055204; L. Ya. Glozman, Phys. Lett. B 575, (2003) 18; B. Jennings and K. Maltman, hep-ph/0308286; C. E. Carlsonet al., Phys. Lett. B579 (2004) 52; C. E. Carlsonet al. Phys.Rev.D70:037501,2004; F. Huanget al., Phys.Lett.B586:69-74,2004; I. M. Narodetskiet al., Phys. Lett. B578, (2004) 318; S. M. Gerasyuta and V. I. Kochkin, hepph/0310225; hep-ph/0310227; R. Bijkeret al., Eur.Phys. J.A22:319-329,2004;hep-ph/0312380.

[8] H. Weigel, Eur. Phys. J. A 2, 391 (1998); Eur. Phys. J. A 21, 133 (2004); H. Walliser and V. B. Kopeliovich, J. Exp. Theor. Phys. 97, 433 (2003) [Zh. Eksp. Teor. Fiz. 124, 483 (2003)].

[9] R. A. Arndt, Ya. I. Azimov, M. V. Polyakov, I. I. Strakovsky and R. L. Workman, Published in Phys. Rev. C69: (2004) 035208 [arXiv: nucl-th/0312126].

[10] D. Diakonov and V. Petrov, Phys. Lett. B 147, 351 (1984); Nucl. Phys. B. 272, 457 (1986); D. Diakonov, V. Petrov and P.V. Pobylitsa, Nucl. Phys. B 306, 809 (1988).

[11] T. Meissner, F. Gruemmer, and K. Goeke, Phys. Lett. B 227296 (1989); H. Reinhardt and R. Wuensch, Phys. Lett. B230, 93 (1989); M. Wakamatsu and H. Yoshiki, Nucl. Phys. A524, 561 (1991). 